\title{
Accurate Predictions of Electron Binding Energies of Dipole-Bound Anions via Quantum Monte Carlo Methods
}

\author{
Hongxia Hao, ${ }^{\dagger}$ James Shee, $\ddagger$ Shiv Upadhyay, ${ }^{\Uparrow}$ Can Ataca, ${ }^{\S}$ Kenneth D. Jordan, \\ and Brenda M. Rubenstein ${ }^{*} \dagger$ \\ $\dagger$ Department of Chemistry, Brown University, Providence, RI 02912 \\ $\ddagger$ Department of Chemistry, Columbia University, New York, NY 10027 \\ \Department of Chemistry, University of Pittsburgh, Pittsburgh, PA 15260 \\ $\S$ Department of Physics, University of Maryland - Baltimore County, Baltimore, MD \\ 21250 \\ E-mail: brenda_rubenstein@brown.edu \\ Phone: (603) 661-2160
}

\begin{abstract}
Neutral molecules with sufficiently large dipole moments can bind electrons in diffuse nonvalence orbitals with most of their charge density far from the nuclei, forming so-called dipolebound anions. Because long-range correlation effects play an important role in the binding of an excess electron and overall binding energies are often only of the order of 10-100s of wave numbers, predictively modeling dipole-bound anions remains a challenge. Here, we demonstrate that quantum Monte Carlo methods can accurately characterize molecular dipole-bound anions with near threshold dipole moments. We also show that correlated sampling Auxiliary Field Quantum Monte Carlo is particularly well-suited for resolving the fine energy differences between the neutral and anionic species. These results shed light on the fundamental limitations of quantum Monte Carlo methods and pave the way toward using them for the study of weakly-bound species that are too large to model using traditional electron structure methods.
\end{abstract}

\section{Graphical TOC Entry}

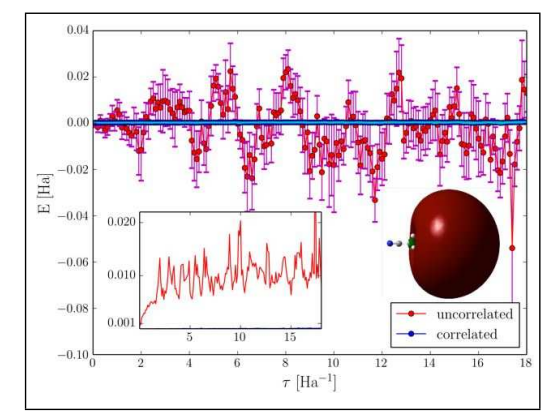

\section{Keywords}

Dipole-Bound, Anions, Electron Binding Energy, Quantum Monte Carlo, Correlated Sampling, Diffusion Monte Carlo, Auxiliary Field Quantum Monte Carlo 
Dipole-bound anions are intriguing species that bind excess electrons via their molecular dipole moments. ${ }^{1,2}$ As the charge-dipole attraction is governed by a long-range potential that behaves as $1 / r^{2}$ at large $r$, dipolebound electrons are delicately bound in diffuse orbitals with most of their charge density located far from the atomic centers of their parent molecules. ${ }^{3-5}$ Within the BornOppenheimer approximation, the critical dipole moment necessary for binding an electron is 1.625 D, ${ }^{6-8}$ but increases to $2.5 \mathrm{D}$ or larger when corrections to the Born-Oppenheimer approximation are made. ${ }^{9-12}$ Beyond being "doorways" to the formation of valence-bound anions, ${ }^{13-16}$ dipole-bound anions may be key contributors to the diffuse interstellar bands, a set of absorption peaks emanating from the interstellar medium whose source has yet to be conclusively identified. ${ }^{17-23}$ The sheer experimental challenge of resolving the exceedingly small binding energies of such fragile species has motivated spectroscopists to produce dipole-bound species via electron attachment ${ }^{24,25}$ and Rydberg electron transfer, ${ }^{12,26,27}$ and to study them via field detachment and photoelectron spectroscopy. ${ }^{28}$ From the theoretical perspective, dipole-bound anions are of particular interest because they pose a formidable challenge for $a b$ initio methods - only high levels of theory, such as coupled cluster theories combined with large, flexible basis sets, are capable of accurately predicting dipole-bound anion electron binding energies that are often of the order of just a few hundred wave numbers in magnitude. ${ }^{2,4,29-32}$ However, these highly accurate methods scale steeply with system size, severely restricting the size of systems to which they can be applied.

Herein, we explore the accuracy with which Diffusion Monte Carlo (DMC) ${ }^{33-35}$ and Auxiliary Field Quantum Monte Carlo (AFQMC), ${ }^{36-41}$ two highly accurate, stochastic methods that scale as only $O\left(N^{3}\right)-O\left(N^{4}\right)$ with system size, can model dipole-bound anions, with the aim of uncovering a new set of approaches for modeling dipole- and correlationbound $^{42}$ anions of large molecules. Interestingly, despite the different approximations they employ, we find that both methods reproduce experimental results and distinguish molecules that bind an extra electron from those that do not. Furthermore, we find that a newly-developed correlated sampling AFQMC approach (C-AFQMC) ${ }^{43}$ is particularly wellsuited for the task of studying energy differences involving weakly-bound species and converges electron binding energies orders of magnitude faster than stochastic methods that do not employ such sampling.

To gauge the viability of characterizing dipole-bound anions using QMC methods, we compute vertical electron affinities for several systems known to form dipole-bound anions. Vertical electron affinities may be obtained by taking the difference between the energies of the neutral and anionic species both calculated at the neutral geometry. However, since the dipole-bound excess electron makes almost no impact on the geometry of the molecule, we can equate these electron affinities to the electron binding energies (EBEs), which are typically defined using the geometry of the anion. In the following paragraphs, we summarize the calculations performed; further details may be found in the Supplemental Information.

In order to compute the EBEs, the neutral geometries were first optimized using the MP2 method $^{44}$ together with the aug-cc-pVDZ basis set ${ }^{45,46}$ in Gaussian 09. ${ }^{47-51}$ Hartree-Fock (HF) wave functions were then generated in Gaussian 09, GAMESS, ${ }^{52,53}$ or NWChem ${ }^{54}$ for use as trial wave functions, which guide sampling and curb the growth of the sign/phase problem, an exponential decay in the signal to noise ratio, in DMC and AFQMC. ${ }^{55,56}$ In order to obtain stable dipole-bound anions, it is essential to use flexible basis sets with very diffuse basis functions. Here, we use the aug-cc-pVDZ basis set augmented with a set of diffuse $s$ and $p$ functions, and in one case, also $d$ functions, located near the positive end of the dipole. ${ }^{4} \mathrm{DMC}^{33,57,58}$ and $\mathrm{AFQMC}^{36,59}$ are performed on all of the species studied. DMC calculations were conducted using the CASINO package. ${ }^{57,60}$ Averages were obtained by sampling the configurations of 4650 walkers for 500,000 or more propagation steps. In order to obtain DMC energies 
in the zero time-step $(\Delta \tau \rightarrow 0)$ limit, DMC simulations were conducted at three different time step sizes and then linearly extrapolated to zero time-step to yield the final values reported.

Because the calculation of dipole-bound anion vertical binding energies involves energy differences between two species with identical molecular geometries, we employed C-AFQMC ${ }^{43}$ for the majority of our AFQMC calculations. In this approach, differences between two quantities normally computed separately using independently generated auxiliary field configurations are instead computed using the same set of configurations. For sufficiently similar systems like many dipole-bound anions and their parent neutral molecules, this can result in a systematic cancellation of errors, which markedly reduces the variance associated with calculated observables. In our calculations, a set of randomly-seeded repeat simulations were initialized, and after an initial equilibration period, the mean and standard error of the cumulative averages were computed among the repeats. Convergence is attained when the mean is visually observed to plateau, and when the statistical error falls below a target threshold. The $\Delta \tau \rightarrow 0$ limit was estimated via linear extrapolations using simulations performed at $\Delta \tau=0.01$ and $\Delta \tau=0.005$ a.u.

While DMC has been successfully employed to calculate cluster binding energies, ${ }^{67,68}$ here we analyze its performance predicting the electron binding energies of molecular dipole-bound anions. As an initial test, we examined whether DMC could faithfully predict whether a given species binds an extra electron or not. Thus, in addition to considering several species known to form dipole-bound anions, we also consider $\mathrm{SO}$, a molecule with a dipole moment of $1.55 \mathrm{D}$, which is below the threshold required for binding. Our results are summarized in Table 1. DMC calculations correctly predicted that all of the species studied, except SO, would form dipole-bound anions.

In Figure 1, the charge densities of neutral $\mathrm{CH}_{3} \mathrm{CN}$, the $\mathrm{CH}_{3} \mathrm{CN}$ anion, and the $\mathrm{SO}$ molecule plus an extra electron are compared. While the DMC charge density for the neutral $\mathrm{CH}_{3} \mathrm{CN}$ molecule is highly localized in the molecular region, that of the anion is far more diffuse and protrudes continuously out from the positive side of the dipole. The charge density plots of the other dipole-bound anions considered in this work manifest similar features and are reported in the Supplemental Information. In stark contrast, the charge density of SO plus an extra electron consists of two disjoint contributions - one associated with the neutral molecule and a second representative of an additional unbound electron positioned more than $50 \AA$ from the molecule.
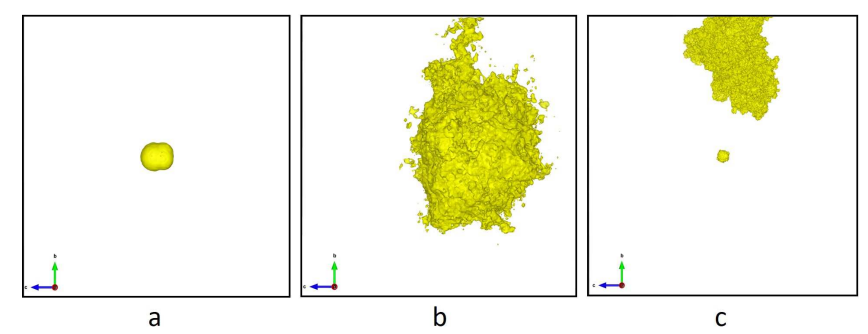

Figure 1: DMC charge densities of (a) neutral $\mathrm{CH}_{3} \mathrm{CN}$, (b) the $\mathrm{CH}_{3} \mathrm{CN}$ anion, and (c) $\mathrm{SO}$ plus an extra electron. The isosurface values taken for each of these plots are $4 \times 10^{-14} \mathrm{e} / \AA^{3}, 4 \times 10^{-14} \mathrm{e} / \AA^{3}$, and $1 \times 10^{-20} \mathrm{e} / \AA^{3}$, respectively. Molecules are placed in the center of the simulation box.

Interestingly, even though the fixed-node error in the energies of the neutral and anion are greater in magnitude than the electron binding energy, DMC calculations using single Slater determinant trial wave functions provide semiqualitatively accurate EBEs of dipole-bound anions. Nevertheless, obtaining quantitatively accurate EBEs with the DMC approach employed here would be too computationally demanding to be practical. As presented in Table 1 , achieving DMC statistical error bars smaller than the binding energies can require hundreds of thousands to millions of DMC iterations, even starting from a well-optimized variational wave function. For example, as depicted in Figure 2, statistical fluctuations in the energy of the $\mathrm{CH}_{3} \mathrm{CN}$ anion simulated with 4650 walkers hover around $65000 \mathrm{~cm}^{-1}$, meaning that over 500,000 samples must be taken to achieve on the order of $100 \mathrm{~cm}^{-1}$ error bars. Thus, while 
Table 1: EBEs and dipole moments of selected species from experiment and SelfConsistent Field [HF], Coupled Cluster $[\mathrm{CCSD}(\mathrm{T})], \mathrm{DMC}$, and C-AFQMC calculations.

\begin{tabular}{|c|c|c|c|c|c|c|}
\hline & \multirow[b]{2}{*}{ Dipole Moment (D) } & \multicolumn{5}{|c|}{ Electron Binding Energy $\left(\mathrm{cm}^{-1}\right)$} \\
\hline & & Experiment & $\Delta \mathbf{S C F}^{a}$ & $\operatorname{CCSD}(\mathrm{T})^{a}$ & $\mathrm{DMC}^{b}$ & C-AFQMC ${ }^{c}$ \\
\hline SO & $1.55^{61}$ & NOT-BOUND & -3.84 & -4.13 & $-308.20 \pm 70.82$ & $-4.54 \pm 0.64$ \\
\hline HCN & $2.98^{61}$ & $13^{62}$ & 11.00 & 7.44 & $46.17 \pm 45.30$ & $10.80 \pm 2.95$ \\
\hline $\mathrm{CH}_{2} \mathbf{C H C N}$ & $3.87^{61}$ & $56-87^{12,63}$ & 43.30 & 61.87 & $106.63 \pm 58.12$ & $65.70 \pm 11.03$ \\
\hline $\mathrm{CH}_{3} \mathrm{CN}$ & $3.92^{61}$ & $93-145^{12,63}$ & 50.83 & 103.00 & $93.83 \pm 36.21$ & $95.85 \pm 9.73$ \\
\hline $\mathbf{C}_{3} \mathbf{H}_{2}$ & $4.14^{64}$ & $170 \pm 50^{65}$ & 54.61 & 162.08 & $151.22 \pm 64.25^{d}$ & $132.45 \pm 9.43^{e}$ \\
\hline $\mathrm{C}_{3} \mathbf{H}_{2} \mathbf{O}_{3}$ & $4.55^{61}$ & $194 \pm 24^{66}$ & 103.13 & 163.31 & $213.98 \pm 116.15$ & $157.70 \pm 17.96$ \\
\hline
\end{tabular}

${ }^{a} \mathrm{HF}$ and CCSD(T) calculations were preformed using Gaussian 09. ${ }^{b}$ DMC calculations of the anion are based on unrestricted Hartree Fock (UHF) trial wave functions obtained from Gaussian 09 or GAMESS. ${ }^{c}$ AFQMC calculations were based on UHF trial wave functions obtained from NWChem. ${ }^{d}$ The DMC calculations on the $\mathrm{C}_{3} \mathrm{H}_{2}$ dipole-bound anion used a restricted open-shell Hartree Fock (ROHF) trial wave function. ${ }^{e}$ To be consistent, the C-AFQMC calculations on the $\mathrm{C}_{3} \mathrm{H}_{2}$ dipole-bound anion were also based on an ROHF trial wave function.

DMC EBEs that agree with experimental measurements and coupled cluster calculations can be gleaned from the noise, it is only with error bars that are still too large to make definitive statements and at great computational expense.

One stochastic technique capable of scaling to dipole-bound anions of large molecules with a substantial reduction in statistical noise is $\mathrm{C}$ AFQMC. As shown in Table 1, C-AFQMC error bars are at least an order of magnitude smaller than DMC error bars using a similar number of samples, but using two orders of magnitude shorter projection time (2000 a.u. was used to obtain most DMC results; 20 a.u. was used to obtain the $\mathrm{C}-\mathrm{AFQMC}$ results). The $\mathrm{C}-\mathrm{AFQMC}$ results presented were moreover obtained starting from just HF wave functions. One might question whether the source of this improvement stems from the AFQMC algorithm or from the use of correlated sampling. Figure 3, which depicts the energy as a function of imaginary projection time for the $\mathrm{CH}_{3} \mathrm{CN}$ molecule, demonstrates that the improvement may be attributed to both sources. Uncorrelated AFQMC simulations are accompanied by statistical fluctuations on the order of $10^{4} \mathrm{~cm}^{-1}$, which are smaller than the $10^{5} \mathrm{~cm}^{-1}$ fluctuations associated with the EBE values from the DMC sim-

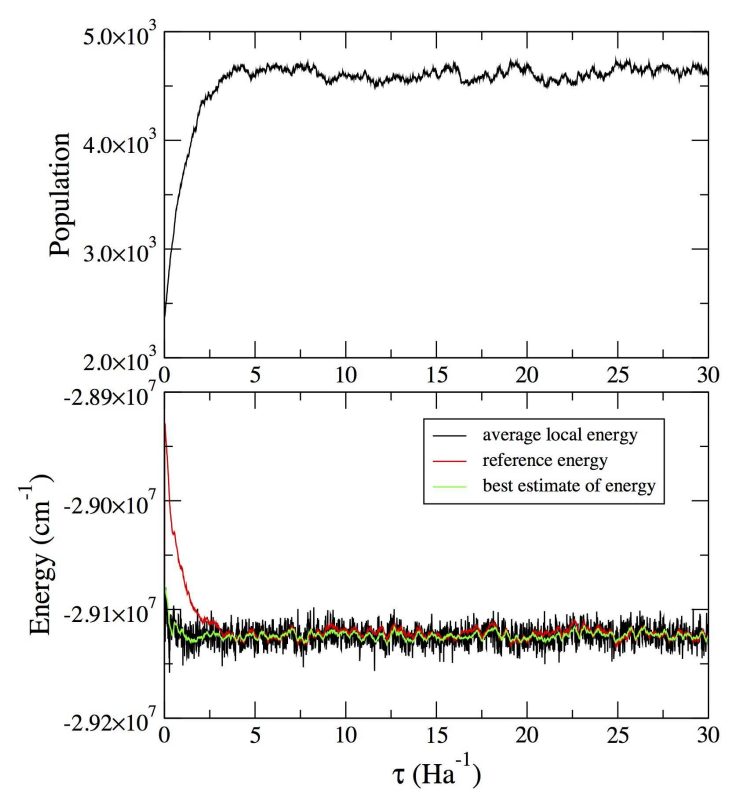

Figure 2: The time evolution of the DMC energy and walker population for the $\mathrm{CH}_{3} \mathrm{CN}$ anion using $\Delta \tau=0.01$ a.u. with 4650 walkers. Walkers were initialized with a UHF trial wave function expanded in terms of the aug-cc-pVDZ basis with a $7 s 7 p$ set of diffuse gaussian-type orbitals. In the Figure, reference energy refers to $E_{T}$ (see Supplemental Information), the average local energy refers to the local energy averaged over walkers at a given imaginary time, and best estimate of the energy refers to the energy averaged over all samples taken up to a certain imaginary time. 


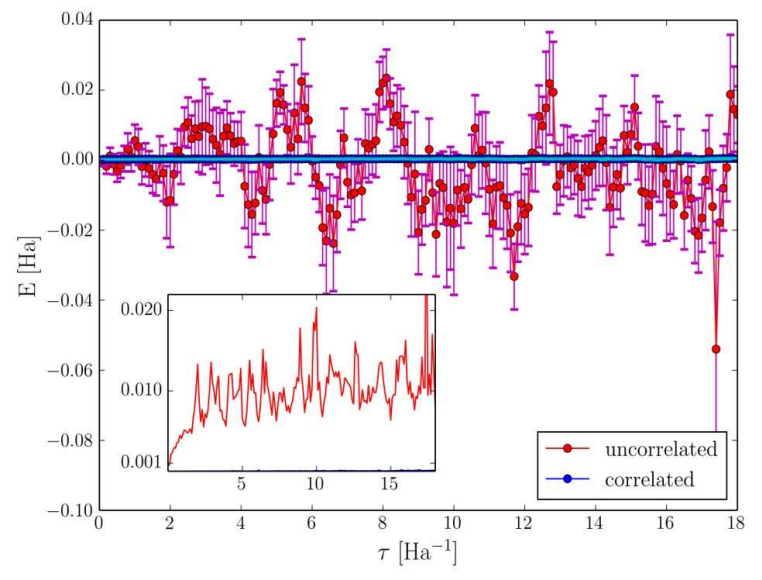

a

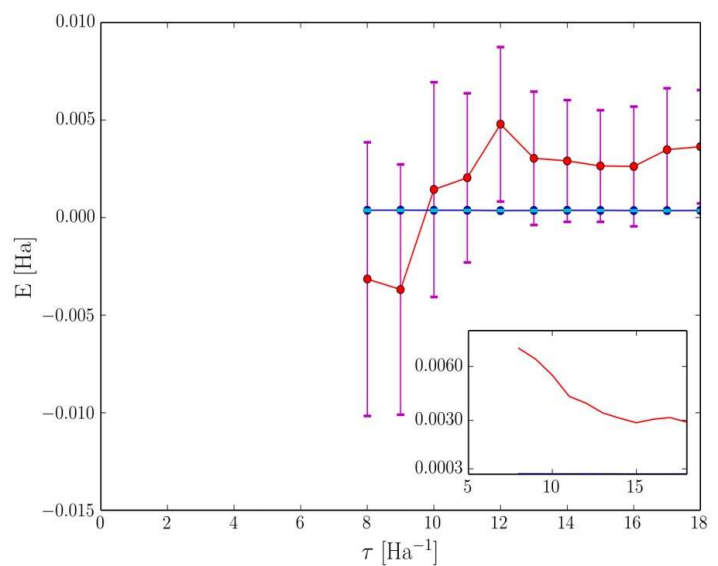

b

Figure 3: Comparison of energies from AFQMC calculations with and without correlated sampling for $\mathrm{CH}_{3} \mathrm{CN}$ using the aug-cc-pvdz $+7 s 7 p$ basis, an HF reference wave function, $\Delta \tau=0.01$ a.u., 384 walkers per simulation, and 17 repeated simulations. (a) Mean values of the EBE (circles) among the repeats at each $\tau$ along the imaginary-time propagation; (b) Mean values of the cumulative averages taken for $\tau>8$ a.u. The error bars give the standard errors, defined as the standard deviation times $\frac{1}{\sqrt{N_{r}}}$, where $N_{r}$ is the number of simulation repeats, and are plotted in the insets for clarity.

ulations (see Figure 2). C-AFQMC calculations are accompanied by almost imperceptible statistical fluctuations on the order of 22 $\mathrm{cm}^{-1}$. Thus, AFQMC's sampling innovations yield meaningful gains above DMC, yet it is the use of correlated sampling that yields, by far, the largest improvements.

As shown in Table 1, C-AFQMC yields energies with sufficiently small error bars that meaningful comparisons can now be made against coupled cluster calculations and experiment. In general, the EBEs predicted by CAFQMC are within error bars of both experimental and previous coupled cluster single, doubles, and perturbative triples $(\mathrm{CCSD}(\mathrm{T}))$ calculations. This demonstrates that phaseless approximation errors are mild and that for dipole-bound anions AFQMC is as accurate as $\operatorname{CCSD}(\mathrm{T})$. The $\mathrm{C}_{3} \mathrm{H}_{2}$ anion, however, stands as one cautionary tale. For the $\mathrm{C}_{3} \mathrm{H}_{2}$ anion, we located two different UHF solutions, neither of which proved suitable for trial functions in QMC calculations (see the Supplemental Information for more details). For this reason, we employed an ROHF trial wave function for the $\mathrm{C}_{3} \mathrm{H}_{2}$ anion instead. This example suggests that care must be taken when selecting trial wave functions for and ultimately simulating larger molecules that may have many compet- ing low-lying states.

In this work, we have demonstrated that lowscaling QMC methods, and in particular, CAFQMC, are capable of resolving the fine energy differences required to accurately predict electron binding energies of dipole-bound anions. Electron binding energies within wave numbers of previous coupled cluster and experimental results were obtained for $\mathrm{HCN}$, $\mathrm{CH}_{2} \mathrm{CHCN}, \mathrm{CH}_{3} \mathrm{CN}, \mathrm{C}_{3} \mathrm{H}_{2}$, and $\mathrm{C}_{3} \mathrm{H}_{2} \mathrm{O}_{3}$. Our results demonstrate that, while uncorrelated DMC and AFQMC methods can qualitatively describe dipole-bound species, only correlated sampling techniques such as C-AFQMC are capable of achieving quantitative accuracy within computationally tractable amounts of time. The success of C-AFQMC in this work beckons for the further development of correlated DMC methods capable of resolving the molecular energy differences that lie at the heart of all chemical processes. These findings pave the way toward using stochastic methods to study the much larger polycyclic aromatic hydrocarbons $(\mathrm{PAH})^{69}$ and long-chain carbon anions ${ }^{70}$ thought to contribute to the diffuse interstellar bands, as well as correlation-bound anions ${ }^{71,72}$ and weakly bound clusters ${ }^{73}$ whose size puts them beyond reach of most high accuracy methods. 
Acknowledgement H.H., J.S., and B.R. acknowledge Lai-Sheng Wang, Yuan Liu, G. Stephen Kocheril, Shiwei Zhang, and David Reichman for their ongoing support and insights. H.H. and B.R. acknowledge support from the U.S. Department of Energy by Lawrence Livermore National Laboratory under Contract DEAC52-07NA27344, 15-ERD-013 and NSF grant DMR-1726213. S.U. and K.D.J. acknowledge the support of NSF grant CHE-1762337. This research was conducted using computational resources and services at the Center for Computation and Visualization, Brown University, the University of Pittsburgh's Center for Research Computing, and the Extreme Science and Engineering Discovery Environment (XSEDE).

\section{References}

(1) Simons, J. Molecular anions. J. Phys. Chem. A 2008, 112, 6401-6511.

(2) Jordan, K. D.; Wang, F. Theory of dipolebound anions. Annu. Rev. Phys. Chem. 2003, 54, 367-396.

(3) Jordan, K. D.; Luken, W. Theoretical study of the binding of an electron to a molecular dipole: $\mathrm{LiCl}^{-}$. J. Chem. Phys. 1976, 64, 2760-2766.

(4) Gutowski, M.; Skurski, P.; Boldyrev, A. I.; Simons, J.; Jordan, K. D. Contribution of electron correlation to the stability of dipole-bound anionic states. Phys. Rev. A 1996, 54, 1906-1909.

(5) Barnett, R. N.; Landman, U.; Cleveland, C. L.; Jortner, J. Electron localization in water clusters. II. Surface and internal states. J. Chem. Phys. 1988, 88, 4429-4447.

(6) Fermi, E.; Teller, E. The capture of negative mesotrons in matter. Phys. Rev. 1947, 72, 399-408.

(7) Turner, J. E.; Anderson, V. E.; Fox, K. Ground-state energy eigenvalues and eigenfunctions for an electron in an electric-dipole field. Phys. Rev. 1968, 174, 81-89.

(8) Crawford, O. H. Bound states of a charged particle in a dipole field. Proc. Phys. Soc. 1967, 91, 279.

(9) Garrett, W. Critical binding of an electron to a non-stationary electric dipole. Chem. Phys. Lett. 1970, 5, $393-397$.

(10) Garrett, W. R. Critical binding of an electron to a rotationally excited dipolar system. Phys. Rev. A 1971, 3, 961-972.

(11) Lykke, K. R.; Mead, R. D.; Lineberger, W. C. Observation of dipole-bound states of negative ions. Phys. Rev. Lett. 1984, 52, 2221-2224.

(12) Desfrançois, C.; Abdoul-Carime, H.; Khelifa, N.; Schermann, J. P. From $\frac{1}{r}$ to $\frac{1}{r^{2}}$ potentials: Electron exchange between Rydberg atoms and polar molecules. Phys. Rev. Lett. 1994, 73, 2436-2439.

(13) Hendricks, J. H.; Lyapustina, S. A.; de Clercq, H. L.; Bowen, K. H. The dipole bound-to-covalent anion transformation in uracil. J. Chem. Phys. 1998, 108, 8-11.

(14) Compton, R. N.; Jr., H. S. C.; Desfrançois, C.; Abdoul-Carime, H.; Schermann, J. P.; Hendricks, J. H.; Lyapustina, S. A.; Bowen, K. H. On the binding of electrons to nitromethane: Dipole and valence bound anions. J. Chem. Phys. 1996, 105, 3472-3478.

(15) Desfrançois, C.; Abdoul-Carime, H.; Schermann, J. P. Electron attachment to isolated nucleic acid bases. J. Chem. Phys. 1996, 104, 7792-7794.

(16) Desfrançois, C.; Periquet, V.; Bouteiller, Y.; Schermann, J. P. Valence and dipole binding of electrons to uracil. J. Phys. Chem. A 1998, 102, 1274-1278.

(17) Sarre, P. J. The diffuse interstellar bands: A dipole-bound state hypothesis. Mon. Not. R. Astron. Soc. 2000, 313, L14-L16. 
(18) Sarre, P. J. The diffuse interstellar bands: A major problem in astronomical spectroscopy. J. Mol. Spectrosc. 2006, 238, 1 -10 .

(19) Güthe, F.; Tulej, M.; Pachkov, M. V.; Maier, J. P. Photodetachment spectrum of $1-\mathrm{C}_{3} \mathrm{H}_{2}^{-}$: The role of dipole bound states for electron attachment in interstellar clouds. ApJ 2001, 555, 466.

(20) Maier, J. P.; Walker, G. A. H.; Bohlender, D. A.; Mazzotti, F. J.; Raghunandan, R.; Fulara, J.; Garkusha, I.; Nagy, A. Identification of $\mathrm{H}_{2} \mathrm{CCC}$ as a diffuse interstellar band carrier. ApJ 2011, 726, 41.

(21) McCall, B. J.; Oka, T.; Thorburn, J.; Hobbs, L. M.; York, D. G. A critical examination of the $\mathrm{l}_{-} \mathrm{C}_{3} \mathrm{H}_{2}{ }^{-}$Spectrum and the diffuse interstellar bands. ApJL 2002, 56 \%, L145.

(22) Fortenberry, R. C.; Crawford, T. D. Theoretical prediction of new dipole-bound singlet states for anions of interstellar interest. J. Chem. Phys. 2011, 134, 154304.

(23) Larsson, M.; Geppert, W. D.; Nyman, G. Ion chemistry in space. Rep. Prog. Phys. 2012, 75, 066901.

(24) Hendricks, J. H.; Lyapustina, S. A.; de Clercq, H. L.; Snodgrass, J. T.; Bowen, K. H. Dipole bound, nucleic acid base anions studied via negative ion photoelectron spectroscopy. J. Chem. Phys. 1996, 104, 7788-7791.

(25) Buytendyk, A. M.; Buonaugurio, A. M.; $\mathrm{Xu}$, S.-J.; Nilles, J. M.; Bowen, K. H.; Kirnosov, N.; Adamowicz, L. Computational and photoelectron spectroscopic study of the dipole-bound anions, indole $\left(\mathrm{H}_{2} \mathrm{O}\right)_{1,2}^{-}$. J. Chem. Phys. 2016, 145, 024301.

(26) Desfrançois, C.; Abdoul-Carime, H.; Schermann, J.-P. Ground state dipolebound anions. Int. J. Mod. Phys. B 1996, 10, 1339-1395.
(27) Hammer, N. I.; Diri, K.; Jordan, K. D.; Desfrançois, C.; Compton, R. N. Dipolebound anions of carbonyl, nitrile, and sulfoxide containing molecules. J. Chem. Phys. 2003, 119, 3650-3660.

(28) Wang, L.-S.; Ding, C.-F.; Wang, X.-B.; Barlow, S. E. Photodetachment photoelectron spectroscopy of multiply charged anions using electrospray ionization. Rev. Sci. Instrum. 1999, 70, 1957-1966.

(29) Gutowski, M.; Skurski, P.; Boldyrev, A. I.; Simons, J.; Jordan, K. D. Contribution of electron correlation to the stability of dipole-bound anionic states. Phys. Rev. A 1996, 54, 1906-1909.

(30) Gutowski, M.; Skurski, P.; Jordan, K. D.; Simons, J. Energies of dipole-bound anionic states. Int. J. Quantum Chem 1997, 64, 183-191.

(31) Nooijen, M.; Bartlett, R. J. Equation of motion coupled cluster method for electron attachment. J. Chem. Phys. 1995, 102, 3629-3647.

(32) Gutsev, G. L.; Nooijen, M.; Bartlett, R. J. Valence and excited dipole-bound states of polar diatomic anions: $\mathrm{LiH}^{-}, \mathrm{LiF}^{-}, \mathrm{LiCl}^{-}$, $\mathrm{NaH}^{-}, \mathrm{NaF}^{-}, \mathrm{NaCl}^{-}, \mathrm{BeO}^{-}$, and $\mathrm{MgO}^{-}$. Chem. Phys. Lett. 1997, 276, $13-19$.

(33) Foulkes, W. M. C.; Mitas, L.; Needs, R. J.; Rajagopal, G. Quantum Monte Carlo simulations of solids. Rev. Mod. Phys. 2001, 73, 33-83.

(34) Morales, M. A.; McMinis, J.; Clark, B. K.; Kim, J.; Scuseria, G. E. Multideterminant wave functions in quantum Monte Carlo. J. Chem. Theory Comput. 2012, 8, 21812188, PMID: 26588949.

(35) Petruzielo, F. R.; Toulouse, J.; Umrigar, C. J. Approaching chemical accuracy with quantum Monte Carlo. J. Chem. Phys. 2012, 136, 124116.

(36) Motta, M.; Zhang, S. Ab initio computations of molecular systems by the 
auxiliary-field quantum Monte Carlo method. WIREs Comput. Mol. Sci. 8, e1364.

(37) Suewattana, M.; Purwanto, W.; Zhang, S.; Krakauer, H.; Walter, E. J. Phaseless auxiliary-field quantum Monte Carlo calculations with plane waves and pseudopotentials: Applications to atoms and molecules. Phys. Rev. B 2007, 75, 245123 .

(38) Purwanto, W.; Zhang, S.; Krakauer, H. An auxiliary-field quantum Monte Carlo study of the chromium dimer. J. Chem. Phys. 2015, 142, 064302.

(39) Al-Saidi, W. A.; Krakauer, H.; Zhang, S. Auxiliary-field quantum Monte Carlo study of $\mathrm{TiO}$ and $\mathrm{MnO}$ molecules. Phys. Rev. B 2006, 73, 075103.

(40) Al-Saidi, W. A.; Krakauer, H.; Zhang, S. Auxiliary-field quantum Monte Carlo study of first- and second-row post-d elements. J. Chem. Phys. 2006, 125, 154110.

(41) Al-Saidi, W. A.; Krakauer, H.; Zhang, S. A study of $\mathrm{H}+\mathrm{H}_{2}$ and several $\mathrm{H}$ bonded molecules by phaseless auxiliaryfield quantum Monte Carlo with plane wave and Gaussian basis sets. J. Chem. Phys. 2007, 126, 194105.

(42) Voora, V. K.; Kairalapova, A.; Sommerfeld, T.; Jordan, K. D. Theoretical approaches for treating non-valence correlation-bound anions. J. Chem. Phys. 2017, 147, 214114.

(43) Shee, J.; Zhang, S.; Reichman, D. R.; Friesner, R. A. Chemical transformations approaching chemical accuracy via correlated sampling in auxiliary-field quantum Monte Carlo. J. Chem. Theory Comput. 2017, 13, 2667-2680.

(44) Møller, C.; Plesset, M. S. Note on an approximation treatment for many-electron systems. Phys. Rev. 1934, 46, 618-622.
(45) Dunning, T. H. Gaussian basis sets for use in correlated molecular calculations. I. The atoms boron through neon and hydrogen. J. Chem. Phys. 1989, 90, 1007-1023.

(46) Kendall, R. A.; Dunning, T. H.; Harrison, R. J. Electron affinities of the firstrow atoms revisited. Systematic basis sets and wave functions. J. Chem. Phys. 1992, 96, 6796-6806.

(47) Frisch, M. J.; Head-Gordon, M.; Pople, J. A. A direct MP2 gradient method. Chem. Phys. Lett. 1990, 166, $275-280$.

(48) Frisch, M. J.; Head-Gordon, M.; Pople, J. A. Semi-direct algorithms for the MP2 energy and gradient. Chem. Phys. Lett. 1990, 166, $281-289$.

(49) Head-Gordon, M.; Pople, J. A.; Frisch, M. J. MP2 energy evaluation by direct methods. Chem. Phys. Lett. 1988, 153, $503-506$.

(50) Saebo, S.; Almöf, J. Avoiding the integral storage bottleneck in LCAO calculations of electron correlation. Chem. Phys. Lett. 1989, 154, $83-89$.

(51) Head-Gordon, M.; Head-Gordon, T. Analytic MP2 frequencies without fifth-order storage. Theory and application to bifurcated hydrogen bonds in the water hexamer. Chem. Phys. Lett. 1994, 220, $122-$ 128.

(52) Schmidt, M. W.; Baldridge, K. K.; Boatz, J. A.; Elbert, S. T.; Gordon, M. S.; Jensen, J. H.; Koseki, S.; Matsunaga, N.; Nguyen, K. A.; Su, S. et al. General atomic and molecular electronic structure system. J. Comput. Chem. 14, 1347-1363.

(53) Gordon, M. S.; Schmidt, M. W. In Theory and applications of computational chemistry; Dykstra, C. E., Frenking, G., Kim, K. S., Scuseria, G. E., Eds.; Elsevier: Amsterdam, 2005; pp 1167 - 1189. 
(54) Valiev, M.; Bylaska, E.; Govind, N.; Kowalski, K.; Straatsma, T.; Dam, H. V.; Wang, D.; Nieplocha, J.; Apra, E.; Windus, T. et al. NWChem: A comprehensive and scalable open-source solution for large scale molecular simulations. Comput. Phys. Commun. 2010, 181, 1477 1489 .

(55) Anderson, J. B. A random-walk simulation of the Schrödinger equation: $\mathrm{H}_{3}^{+}$. J. Chem. Phys. 1975, 63, 1499-1503.

(56) Ceperley, D. Fermion nodes. J. Stat. Phys. 1991, 63, 1237.

(57) Needs, R. J.; Towler, M. D.; Drummond, N. D.; Ríos, P. L. Continuum variational and diffusion quantum Monte Carlo calculations. J. Phys.: Condens. Matter 2010, 22, 023201.

(58) Toulouse, J.; Assaraf, R.; Umrigar, C. J. In Electron correlation in molecules - ab initio beyond Gaussian quantum chemistry; Hoggan, P. E., Ozdogan, T., Eds.; Advances in quantum chemistry; Academic Press, 2016; Vol. 73; pp 285 - 314.

(59) Zhang, S. Emergent phenomena in correlated matter: Modeling and simulation; 2013; Vol. 3; Chapter Auxiliary-field quantum Monte Carlo for correlated electron systems.

(60) Drummond, N. D.; Needs, R. J. Varianceminimization scheme for optimizing Jastrow factors. Phys. Rev. B 2005, 72, 085124 .

(61) Nelson Jr, R. D.; Lide Jr, D. R.; Maryott, A. A. Selected values of electric dipole moments for molecules in the gas phase; 1967.

(62) Ard, S.; Garrett, W. R.; Compton, R. N.; Adamowicz, L.; Stepanian, S. G. Rotational states of dipole-bound anions of hydrogen cyanide. Chem. Phys. Lett. 2009, 473, 223-226.
(63) Desfrançois, C. Determination of electron binding energies of ground-state dipolebound molecular anions. Phys. Rev. A 1995, 51, 3667-3675.

(64) Gottlieb, C. A.; Killian, T. C.; Thaddeus, P.; Botschwina, P.; Flügge, J.; Oswald, M. Structure of propadienylidene, $\mathrm{H}_{2}$ CCC. J. Chem. Phys. 1993, 98, 44784485 .

(65) Yokoyama, K.; Leach, G. W.; Kim, J. B.; Lineberger, W. C.; Boldyrev, A. I.; Gutowski, M. Autodetachment spectroscopy and dynamics of vibrationally excited dipole-bound states of $\mathrm{H}_{2} \mathrm{CCC}^{-}$. J. Chem. Phys. 1996, 105, 10706-10718.

(66) Hammer, N. I.; Hinde, R. J.; Compton, R. N.; Diri, K.; Jordan, K. D.; Radisic, D.; Stokes, S. T.; Bowen, K. H. Dipole-bound anions of highly polar molecules: ethylene carbonate and vinylene carbonate. J. Chem. Phys. 2004, 120, 685-690.

(67) Xu, J.; Jordan, K. D. Application of the diffusion Monte Carlo method to the binding of excess electrons to water clusters. J. Phys. Chem. A 2010, 114, 1364-1366, PMID: 19788288.

(68) Wang, F.-F.; Deible, M. J.; Jordan, K. D. Benchmark study of the interaction energy for an $\left(\mathrm{H}_{2} \mathrm{O}\right)_{16}$ cluster: Quantum Monte Carlo and complete basis set limit MP2 results. J. Phys. Chem. A 2013, 117, 7606-7611, PMID: 23763299.

(69) Voora, V. K.; Jordan, K. D. Nonvalence correlation-bound anion states of polycyclic aromatic hydrocarbons. J. Phys. Chem. Lett 2015, 6, 3994-3997.

(70) Tulej, M.; Kirkwood, D. A.; Pachkov, M.; Maier, J. P. Gas-phase electronic transitions of carbon chain anions coinciding with diffuse interstellar bands. ApJL 1998, 506, L69.

(71) Voora, V. K.; Cederbaum, L. S.; Jordan, K. D. Existence of a correlation 
bound s-type anion state of $\mathrm{C}_{60}$. J. Phys. Chem. Lett 2013, 4, 849-853.

(72) Voora, V. K.; Kairalapova, A.; Sommerfeld, T.; Jordan, K. D. Theoretical approaches for treating non-valence correlation-bound anions. J. Chem. Phys. 2017, 147, 214114.

(73) Zen, A.; Brandenburg, J. G.; Klimeš, J.; Tkatchenko, A.; Alfè, D.; Michaelides, A. Fast and accurate quantum Monte Carlo for molecular crystals. Proc. Natl. Acad. Sci. 2018, 